\title{
Procesos de autoevaluación en la Escuela Interamericana de Bibliotecología de la Universidad de Antioquia, en clave de investigación
}

\author{
María Teresa Múnera Torres \\ Orlanda Jaramillo * \\ José Daniel Moncada Patiño **
}

Artículo recibido:

9 de mayo de 2013.

Artículo aceptado:

7 de octubre de 2013.

\section{Resumen}

El artículo da cuenta del proceso de Autoevaluación (2011), con fines de renovación de la Acreditación de Alta Calidad, que se desarrolló en la Escuela Interamericana de Bibliotecología de la Universidad de Antioquia, como una dinámica de orden investigativo, que pueda servir como referente a experiencias similares.

Palabras clave: Acreditación, Autoevaluación, Ciencia de la Información, Bibliotecología, Aseguramiento de la Calidad.

* Las dos autoras pertenecen a la Universidad de Antioquia, Colombia. (María: mmuner@bibliotecologia.udea.edu.co); (Orlanda: ojara@bibliotecologia.udea.edu.co) ** Ministerio de Cultura-Biblioteca Nacional de Colombia. jdmoncada@mincultura gov.co

INVESTIGACIÓN BIBLIOTECOLÓGICA, Vol. 28, Núm.62, enero/abril, 2014, México, ISSN: 0187-358X. pp. 123-141 


\section{Abstract}

Self-assessment processes as objects of research in the Inter-American School of Librarianship at the University of Antioquia

Maria-Teresa Múnera-Torres, Orlanda Jaramillo and José -Daniel Moncada-Patiño

The article gives an account of the self-assessment process for the purposes of renewal of the High Quality Certificate carried out in 2011as a research activity in the Inter-American School of Librarianship at the University of Antioquia, which might serve as a benchmark for other institutions involved in similar activities.

Keywords: Accreditation; Self-Assessment; Science Information; Library Science; Quality Assurance.

\section{INTRODUCCIÓN}

T os procesos de autoevaluación que acreditan los programas de educación superior se constituyen en jalonadores de acciones innovadoras y de mejoramiento continuo, además de ser una excelente oportunidad para que la institución reafirme su compromiso con el cambio, guiada por su decisión de revisar su estado y proyectar su futuro para continuar siendo una institución socialmente legítima y referente de la educación superior en el área de la Ciencia de la Información en el ámbito local, nacional e internacional.

De esta manera los procesos de autoevaluación y acreditación permiten constatar y mantener el respaldo social y estatal, a la vez que facilitan el diálogo nacional e internacional con otros programas y universidades pares que generan conocimiento de alta exigencia, ya que acreditarse significa hacerse merecedor al crédito, lo que se traduce en ganar o ratificar la confianza a través de las evidencias de tener calidad y creer en ella.

A continuación se presentan los aspectos más destacados del desarrollo del último proceso de autoevaluación con fines de acreditación realizado por la Escuela Interamericana de Bibliotecología (EIB), dentro del contexto de una dinámica investigativa que describe una problemática a resolver, una metodología de indagación, unos análisis de información recopilada, así como los resultados de las estrategias de medición y valoración de la calidad del programa 
de Bibliotecología de la Universidad de Antioquia, a la luz de la normatividad que orienta el gobierno colombiano, para culminar con las correspondientes conclusiones y recomendaciones que se generan como fruto de dicho proceso. Del texto hace parte la Resolución 14957 (19 de noviembre de 2012) del Ministerio de Educación Nacional, la cual recoge las recomendaciones del Consejo Nacional de Acreditación al Programa.

\section{Metodología}

La metodología que se desarrolla en los procesos de acreditación está enmarcada dentro de los lineamientos que se emiten desde el Consejo Nacional de Acreditación (CNA), entidad adscrita al Ministerio de Educación Nacional de Colombia (MEN), y que en términos generales se manifiestan a través de tres etapas: la autoevaluación, la evaluación por pares externos y la evaluación final realizada por el CNA con base en la información que se genera en las dos primeras etapas.

\section{Planteamiento del problema}

La calidad en la formación superior es uno de los aspectos que mayor relevancia cobra al momento de confrontar el desempeño de un profesional con el cúmulo de conocimientos y competencias desarrollados durante su proceso de formación, ya que precisamente con la adecuada y eficiente preparación se logran estructurar perfiles cuya proyección a la sociedad se ofrece de manera adecuada y exitosa. No obstante, algunos ámbitos del desempeño profesional manifiestan limitaciones y aspectos susceptibles de ser mejorados. En consecuencia, es necesario llevar a cabo una dinámica que permita estudiar diferentes aspectos que influyan positiva o negativamente en el proceso de formación de determinados profesionales. En tal sentido, los gobiernos de diferentes países del mundo han generado y promocionado dinámicas de revisión, autorregulación o autoevaluación tanto de las universidades como de sus correspondientes programas académicos. Una de dichas dinámicas es la definición de protocolos de autoevaluación con miras a alcanzar un nivel alto de acreditación y de prestigio ante la sociedad. En este documento se pretende dar a conocer la última experiencia de autoevaluación que se llevó a cabo en la Escuela Interamericana de Bibliotecología de la Universidad de Antioquia, con la intención de lograr la Acreditación de Alta Calidad en el programa de Bibliotecología. 


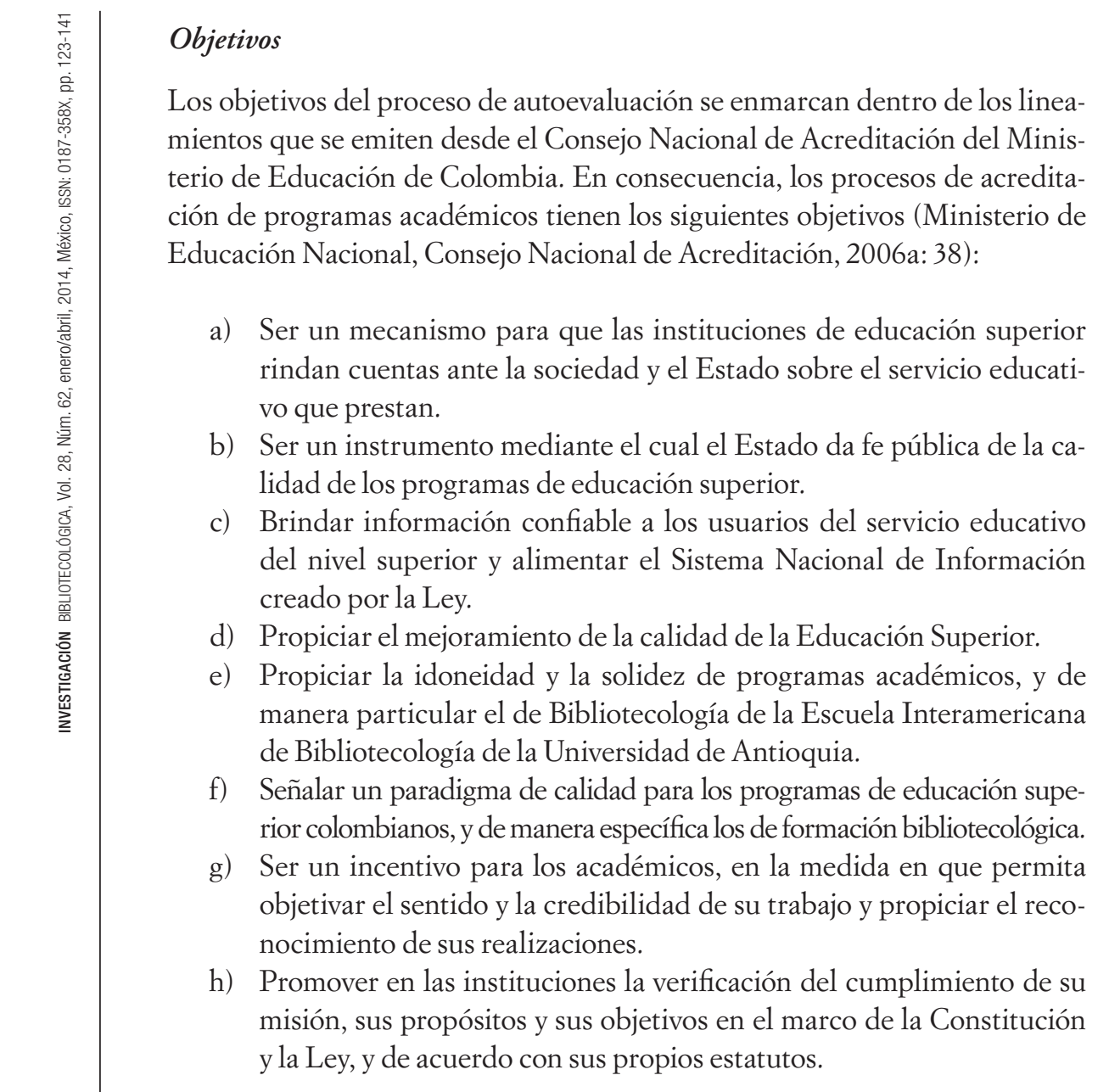

\section{Proceso metodológico de LA ACReditación}

Del proceso de acreditación hacen parte las etapas de autoevaluación, la evaluación externa y la evaluación final, cuyo desarrollo se manifiesta de manera secuencial, como se precisa a continuación.

\section{Etapa de autoevaluación}

La autoevaluación, en el marco de la acreditación de alta calidad, se constituye en un importante instrumento de reflexión y apoyo para revisar el grado de calidad 
con el que cuenta una institución de educación superior o un programa académico en particular. En ella se realiza la evaluación de la universidad o un programa específico por la misma institución, con base en los criterios definidos por el organismo que coordina los procesos de aseguramiento de la calidad en cada país. Para el caso de Colombia es el Consejo Nacional de Acreditación, que a su vez depende del Ministerio de Educación Nacional. Los criterios de autoevaluación se expresan en factores, características e indicadores (MEN/CNA, 2006a: 49).

Cada institución debe desarrollar el proceso de autoevaluación con la participación y el compromiso de los estamentos que la conforman; es decir, estudiantes, docentes, personal administrativo, directivos, egresados y empleadores. Además se deben llevar a cabo las etapas siguientes:

a) Conformación del Comité de Autoevaluación,

b) Diseño y desarrollo metodológico del proceso y

c) Análisis e interpretación de los resultados y elaboración de informe final del proceso.

\section{Etapa de evaluación externa o evaluación por pares}

En esta etapa se utiliza como insumo fundamental el informe de autoevaluación, con base en el cual se verifican sus resultados, se identifican las condiciones internas de operación de la universidad o del programa académico objeto de estudio y se concluye con un juicio sobre la calidad de la universidad o sobre el programa específico.

Esta actividad es responsabilidad de los pares externos, quienes a partir del informe de autoevaluación identifican las condiciones internas de operación de la universidad o programa, constatan y verifican los datos, comparan la documentación e información recopilada de manera directa con las audiencias (docentes, directivos, estudiantes, egresados y empleadores) y concluyen con el juicio sobre la calidad, que plasman en el informe de los pares académicos ante el organismo autorizado en cada país para coordinar los procesos de aseguramiento de la calidad. Para el caso de Colombia, es el CNA. Este proceso se realiza con base en los lineamientos para la acreditación de programas y dentro del marco de la normatividad que rige los procesos de acreditación de programas de instituciones acreditadas.

\section{Etapa de evaluación final}

En esta etapa se lleva a cabo la evaluación final por parte del organismo coordinador de los procesos de aseguramiento de la calidad en cada país, el Consejo 
Nacional de Acreditación para Colombia, y los resultados de la autoevaluación y de la evaluación externa.

Con la información enviada al CNA por los pares externos, quienes dan un juicio valorativo sobre la calidad del programa y recomendaciones para el mejoramiento del mismo, el CNA valora dicha evaluación y emite su juicio sobre el programa al Ministerio de Educación Nacional, organismo que refrenda dicho juicio valorativo en años de acreditación de alta calidad.

\section{Alcance}

El proceso de autoevaluación con fines de acreditación, del cual se da cuenta en el presente informe, es la última experiencia que se lleva a cabo con el Programa de Bibliotecología de la Escuela Interamericana de Bibliotecología de la Universidad de Antioquia y cuyo periodo objeto de autoevaluación es el comprendido entre noviembre de 2004 y noviembre de 2010.

\section{Proceso de autoevaluación de la EIB}

Para el caso de la Escuela Interamericana de Bibliotecología de la Universidad de Antioquia, la autoevaluación es la actividad mediante la cual esta Unidad Académica analiza y examina el estado y la calidad de uno de sus programas académicos, el Programa de Bibliotecología. Se trata de la principal estrategia para explorar sus compromisos y procedimientos y para conocer las respuestas que permitan saber si el programa cumple sus propósitos, objetivos y misión con pertinencia, calidad, voluntad de cambio continuo, y se proyecte como una profesión socialmente viable. Para lograr lo propuesto, la Escuela desarrolla el proceso de autoevaluación con la participación y el compromiso de todos sus estamentos (estudiantes, profesores, egresados, personal administrativo, directivos y empleadores), con el fin de someter sus resultados a la mirada de pares académicos para que éstos conceptúen sobre el estado y la calidad del programa de Bibliotecología.

En la Escuela Interamericana de Bibliotecología de la Universidad de Antioquia el proceso de autoevaluación se realiza en tres fases (Universidad de Antioquia Escuela Interamericana de Bibliotecología, 2011: 40):

1. Conformación del Comité.

2. Diseño metodológico del proceso.

3. Resultados del proceso. 


\section{Conformación del Comité}

El Comité Coordinador se conforma por tres profesores con experiencia en la EIB y en los procesos de autoevaluación con fines de acreditación. Su propósito es consolidar la evaluación como un componente constitutivo del programa de Bibliotecología desde una metodología participativa y en comunicación con los actores involucrados en el proceso evaluativo.

Con el fin de contar con un instrumento que sirva de carta de navegación para el desarrollo de esta actividad, el Comité diseña el plan de acción del proceso de autoevaluación, plan que contempla las tareas, los tiempos y los responsables. Este plan es revisado y ajustado permanentemente. Además, se socializa con todos los estamentos que hacen parte de la comunidad académica: profesores, estudiantes, personal administrativo, egresados y empleadores. Este plan de acción se constituye en la herramienta fundamental para orientar y regular dicho proceso y su labor central se basa en la confrontación de la información hallada con el contexto y la realidad del Programa, por consiguiente la principal actividad se enfoca en el constante análisis de la información y su comunicación a la comunidad académica.

- Revisión y análisis de la documentación existente Luego de elaborar el plan de acción, el Comité asume como tarea esencial la revisión y análisis de la información consignada en fuentes primarias y secundarias, la cual se lleva a cabo en forma exhaustiva, con rigor y objetividad, factores esenciales en el proceso. De esta manera se hace un acercamiento a la información institucional producida durante el periodo de vigencia de la acreditación, en el último caso, 2003 2010; además de revisar la información sobre la EIB existente en los archivos de la Universidad de Antioquia.

- Sensibilización de la comunidad académica

Para la sensibilización y divulgación del proceso, se emplean las siguientes estrategias y medios de comunicación: carteleras, charlas de motivación y aprestamiento (en las asignaturas del plan de estudios), correo electrónico, página web de la Escuela, reuniones informativas, boletín electrónico de la Escuela y de egresados, redes sociales y la lista de discusión Biblio EIB.

\section{Diseño metodológico del proceso}

El proceso de autoevaluación se diseña y desarrolla con un enfoque cualitativo-interpretativo que emplea el método de estudio de casos, se utilizan las 


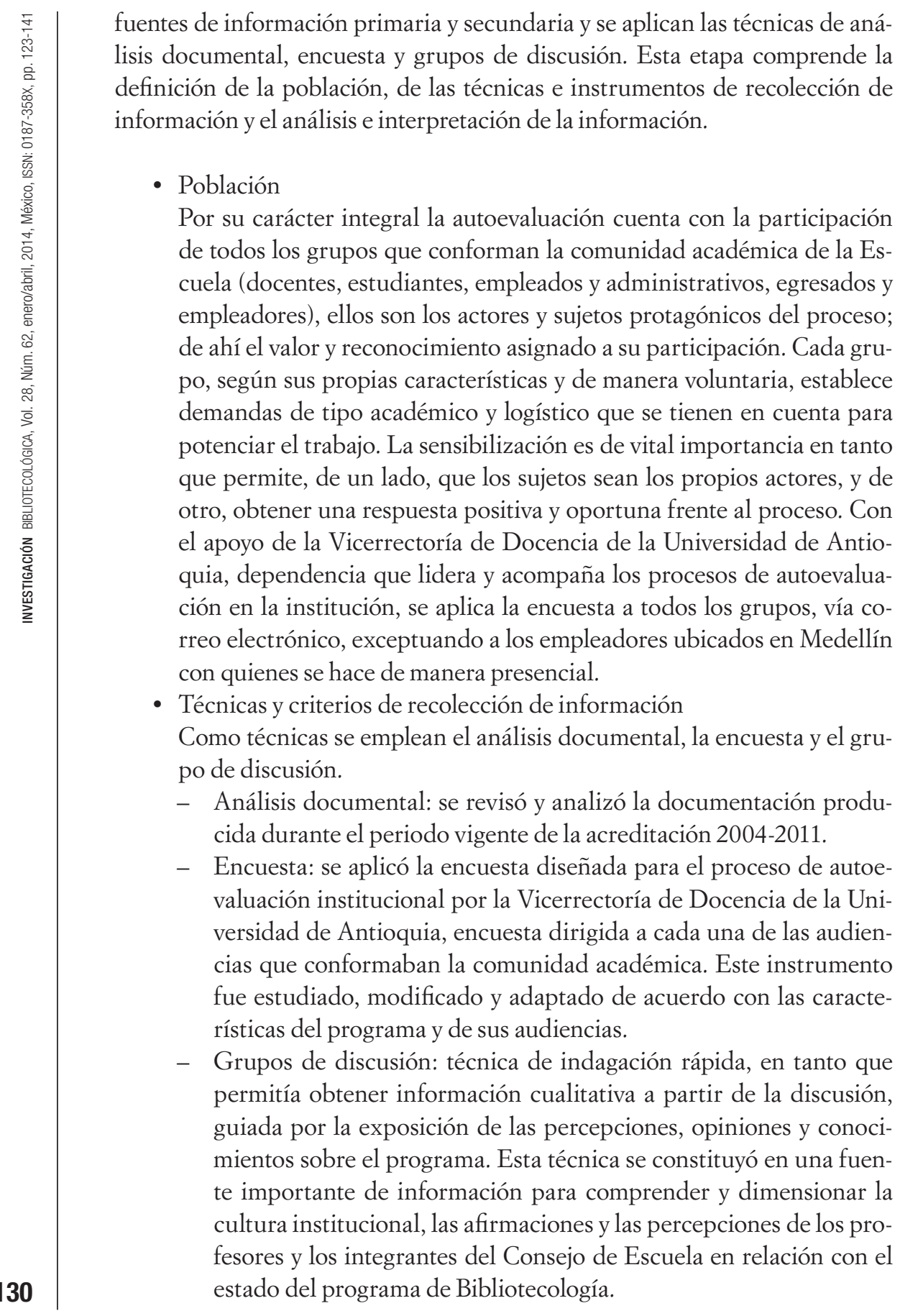


- Criterios para la aplicación de la encuesta

Estudiantes: el criterio de selección fue el total de créditos cursados por el estudiante, definiendo como mínimo 20 créditos (el total de créditos del Programa es de 144), pues con ello se garantizaba que el estudiante tuviera una permanencia mínima de un semestre académico en el Programa; en total se enviaron 291 encuestas.

Docentes: se integraron al proceso todos los profesores vinculados a la Escuela (planta, ocasionales y de cátedra). En total se enviaron 41 encuestas.

Personal administrativo y empleados: se les aplicó la encuesta a los empleados vinculados al programa de Bibliotecología durante el periodo 20042011, durante un año o más, para un total de 13 encuestas aplicadas.

Egresados: para la selección de esta audiencia se consideraron todos los egresados registrados en la base de datos de la EIB que se graduaron después del año 2000 y aquellos que participaron en las actividades que la Escuela realizó durante el periodo 2004-2010. El total de encuestas enviadas vía correo electrónico fue de 238.

Empleadores: se seleccionaron las instituciones con alta demanda de los egresados de la EIB y aquéllas con las que la Institución mantenía contratos de asesoría y consultoría.

Dadas las condiciones de facilidad de aplicación que ofrecen las tecnologías de la información y el sistema de comunicaciones de la Universidad de Antioquia, la encuesta se envió por correo electrónico a todas las audiencias, excepto a los empleadores de Medellín, Bogotá y Cartagena, a quienes se aplicó de manera personalizada. La Tabla 1 recoge el total de la muestra y la tasa de retorno.

Tabla 1. Análisis, interpretación de la información y resultados del proceso.

\begin{tabular}{|l|c|c|c|}
\hline \multicolumn{1}{|c|}{ Audiencias } & Universo de la población & Total de respuestas & Retorno \\
\hline Estudiantes & 291 & 248 & $60 \%$ \\
\hline Docentes & 41 & 27 & $67 \%$ \\
\hline Administrativos & 13 & 11 & $90 \%$ \\
\hline Egresados & 238 & 67 & $30 \%$ \\
\hline Empleadores & 23 & 22 & $90 \%$ \\
\hline
\end{tabular}

Una vez obtenida la información producto de la revisión documental y de la aplicación de la encuesta, se procedió a su análisis e interpretación.

- Definición de categorías y subcategorías

Para efectos del análisis de información se asumieron como categorías y subcategorías de análisis los ocho factores establecidos por el CNA 
para los procesos de autoevaluación de programas de Instituciones de Educación Superior (IES): Proyecto Educativo Institucional (PEI), estudiantes, profesores, procesos académicos, bienestar institucional, egresados, organización, administración y gestión y recursos físicos y financieros; con sus 42 características y 152 indicadores. A partir de ellos se diseñaron y aplicaron las encuestas a las diferentes audiencias, se estructuraron los grupos de discusión y se efectuó el análisis de la información (cf. MNE/CNA, 2006a).

- Análisis de variables

Una vez efectuadas las encuestas se procedió a comparar las respuestas de las audiencias en sus categorías comunes para establecer variables de análisis. Estas variables permitieron observar las recurrencias y las diferencias en las respuestas, que apoyaron el análisis descriptivo de la información recabada sobre el programa.

- Análisis descriptivo

Ya definidas las categorías y subcategorías se procedió a un análisis descriptivo de cada uno de los factores del proceso de autoevaluación. Este análisis se caracterizó por estar construido a partir de la voz de los actores, de sus percepciones, sentires y opiniones sobre los diferentes procesos que analiza el informe. A partir del aplicativo de encuestas diseñado por la Vicerrectoría de Docencia de la Universidad de Antioquia, se obtuvieron gráficos de las respuestas de las audiencias a partir de las cuales se realizó un extenso texto descriptivo y explicativo de las categorías y las tendencias.

- Validación y triangulación de la información

$\mathrm{Al}$ análisis descriptivo le siguió un proceso de validación y confrontación de los resultados con las audiencias, en reuniones y grupos de discusión con los profesores y el Consejo de Escuela. Este momento se caracterizó por ser un proceso participativo en el cual los diferentes estamentos conocieron la interpretación de sus opiniones, construida a partir de las encuestas, y tuvieron la oportunidad de comparar, analizar y hacer ajustes a sus visiones y percepciones.

- Ponderación de factores, características e indicadores

El Comité de Autoevaluación realizó la ponderación de los factores de acuerdo con los siguientes criterios:

1. Lineamientos del documento Ponderación de factores y características para la autoevaluación, MEN/CNA, 2006, que clasifica los factores en tres grupos: el grupo uno incluye proyecto institucional, procesos académicos, profesores, estudiantes y egresados; el grupo dos contempla organización, administración y gestión, recursos 
físicos y financieros, y el grupo tres considera el bienestar institucional.

2. Plan de Mejoramiento y resultados del proceso de autoevaluación 2003. Se le asigna mayor peso a los factores donde el programa presenta debilidades.

3. La consideración de los procesos académicos como la razón de ser del programa.

Como resultado de la aplicación de estos criterios, la ponderación de los factores presenta el siguiente peso:

Tabla 2. Ponderación de factores

\begin{tabular}{|c|c|}
\hline Factores & Puntos de ponderación \\
\hline Proyecto institucional y proyecto educativo & 15 \\
\hline Estudiantes & 15 \\
\hline Profesores & 15 \\
\hline Procesos académicos & 20 \\
\hline Bienestar institucional & 10 \\
\hline Administración, organización y gestión & 7.5 \\
\hline Egresados y articulación con el medio & 10 \\
\hline Recursos físicos y financieros & 7.5 \\
\hline Total & 100 \\
\hline
\end{tabular}

- Características

El Comité asumió las 42 características contenidas en la Guía de autoevaluación de la Universidad de Antioquia (2006). La ponderación de estas características correspondió a la sumatoria de la ponderación de los indicadores correspondientes a cada una.

- Indicadores

De acuerdo con las particularidades del programa, el Comité asumió 152 de los 183 indicadores expuestos en la Guía de autoevaluación. Para su ponderación se contó con la participación de los profesores adscritos al programa de Bibliotecología y el cuerpo administrativo de la Escuela. En correspondencia con las fortalezas y la experiencia de la planta docente y administrativa, el Comité asignó los indicadores de cada factor a un grupo de tres profesores para que los calificaran en un rango de 1 a 5 cada indicador, según su prioridad e importancia en el programa de Bibliotecología. La asignación de tres calificadores tuvo como finalidad asegurar mayor objetividad en la valoración.

- Valoración y cuantificación de los indicadores y características Una vez realizado el análisis descriptivo de los factores y su validación por las respectivas audiencias y por el Consejo de Escuela, se procedió 
a calificar los indicadores y características evaluadas. En este proceso de calificación se tiene en cuenta el peso definido para las características del proceso y la valoración (ponderación) establecida para cada indicador por los docentes del programa en sus áreas de especialidad. Los indicadores de percepción y características se calificaron en una escala del 1 al 5 .

A partir del puntaje de cada factor y de la valoración de cada indicador es posible definir el peso de cada característica según su grupo de indicadores. Una vez obtenida esta valoración el Comité procedió a definir la determinación e importancia de cada característica dentro del proceso de análisis de la información. La calificación final se aplicó a los tres grupos definidos para la valoración y ponderación, de acuerdo con los porcentajes obtenidos en la siguiente escala cualitativa: A. Se cumple plenamente, B. Se cumple en alto grado, C. Se cumple aceptablemente, D. Se cumple insatisfactoriamente, E. No se cumple.

\section{Resultados del proceso}

El proceso de autoevaluación del programa de pregrado en Bibliotecología se centró en la revisión del Plan de Mejoramiento 2003-2007, a la luz de las fortalezas y debilidades encontradas por el CNA; en la comparación entre los documentos que rigen el quehacer de la institución y los resultados de la consulta a las audiencias que conforman la comunidad académica de la EIB, y en la ponderación de los factores, la valoración de las características y en los indicadores propuestos por el CNA.

En consecuencia, al analizar cada uno de los indicadores (152 en total) asignados a las 42 características que estableció el CNA para evaluar la calidad del programa de Bibliotecología, se evidenció que existen las condiciones que demuestran su calidad, tal como lo constata la calificación de cada uno de los ocho factores que a continuación se resaltan.

Proyecto Educativo Institucional. La Escuela tiene formulado su PEI, pero es necesario actualizarlo a la luz de los nuevos programas académicos y los cambios curriculares de los últimos años. Durante el periodo evaluado se cumplieron dos mejoramientos curriculares, lo que demuestra la constante evaluación del currículo.

Profesores. La planta de profesores se ha venido cualificando. A la fecha, la totalidad de los profesores cuentan con título de magister y doctorado o son candidatos a obtenerlo. También sobresale la interdisciplinariedad, la Escuela cuenta con docentes con formación en diferentes áreas afines a la Bibliotecología; la producción académica y participación de los profesores en eventos académicos 
como ponentes va en continuo ascenso, mostrando avances y visibilidad nacional e internacional con la producción intelectual de la EIB.

Estudiantes. Se destaca su incursión en las TIC como parte integral de su formación y perfil profesional. En la investigación sobresalen las modalidades de Estudiantes en Formación, Auxiliares de Investigación y Jóvenes Investigadores, y en especial la interacción y comunicación permanente con los profesores, el personal administrativo y entre ellos, como comunidad estudiantil. Cabe resaltar que, aunque un porcentaje alto de los estudiantes ingresaron a la Escuela como segunda opción, en el transcurso de la carrera van incrementando su sentido de pertenencia y perfilando sus intereses y motivaciones hacia la profesión.

Procesos académicos. Durante el periodo evaluado se realizaron procesos de mejoramiento curricular; producto de estos procesos es la versión curricular cuatro, de favorable aceptación por parte de estudiantes, profesores y sectores externos a la institución. Cambios que, por lo que implican de impacto en la comunidad, han favorecido la reflexión frente a la fundamentación de la profesión y el papel del profesional de la información en la sociedad. A los mejoramientos del currículo se suma la creación de nuevos programas, en especial el inicio de la nueva cohorte de la Maestría en Ciencia de la Información, la Especialización en Edición de Publicaciones y la creación del pregrado de Tecnología en Archivística, también fruto de desarrollo del programa de Bibliotecología por cuanto dan cuenta del análisis sobre la profesión y sus relaciones, gracias a lo cual la Escuela ha decidido promover la fortaleza disciplinar con pregrados separados y la integración investigativa con su posgrado, que agrupa todas las líneas de las llamadas ciencias de la información.

Bienestar institucional. Los programas y actividades que lleva a cabo la Escuela están orientados a brindar un ambiente propicio para la consolidación de la comunidad académica, la formación integral del estudiante y a reducir los factores de deserción. Para alcanzar este propósito, la Coordinación de Bienestar de la Escuela realizó actividades de carácter educativo, cultural, recreativo y de apoyo integral en educación, salud física y mental y estímulos financieros, actividades que posibilitan un ambiente propicio para el desarrollo de las actividades de toda la comunidad.

Administración, organización y gestión. La Escuela destaca como uno de sus logros más significativos la administración del currículo, que cuenta con una favorabilidad alta por parte de toda la comunidad académica en cuanto al desempeño de la Jefatura de Formación Académica, al igual que el reconocimiento de la calidad y eficiencia de la Dirección y el personal administrativo de apoyo.

Egresados y su impacto en el medio. La extensión reviste suma importancia para la Escuela; es a partir de ella que se establece contacto con el medio, lo 
que permite proyectar la academia a la sociedad y conocer la realidad del medio laboral e identificar las necesidades y demandas existentes en el campo donde se desempeñan sus egresados, tanto para el mejoramiento curricular permanente como para el acceso a los servicios de asesoría y consultoría. La valoración de la interacción de la Escuela con sus egresados y el impacto de las actividades de actualización profesional son elementos que deben mejorar, según los resultados del proceso.

Recursos físicos y financieros. La existencia de una institución educativa de nivel universitario requiere una adecuada gestión de su presupuesto para su funcionamiento. Al respecto, es notoria la inversión que la Escuela ha realizado en los últimos años para adecuar de la planta física, la dotación de aulas, la adquisición de equipos, mobiliario y recursos informáticos, y también para apoyar la participación de los estamentos en eventos académicos, todos estos aspectos determinantes para el buen desarrollo del programa. Un importante porcentaje de estos recursos corresponde a la oferta de servicios y de actividades de extensión universitaria.

\section{Juicio global sobre el programa}

La revisión del Plan de Mejoramiento 2003-2007, a la luz de las fortalezas y debilidades encontradas por el CNA, la comparación entre los documentos que rigen el quehacer de la institución y los resultados de la consulta a las audiencias que conforman la comunidad académica de la EIB, permite afirmar que ha cumplido las metas trazadas y que está respondiendo satisfactoriamente a la sociedad, además de desarrollar acciones tendientes a aumentar su calidad y pertinencia social, como lo demuestra la renovación del registro calificado y los programas de Bibliotecología, Tecnología en Archivística, Especialización en Gerencia de Servicios de Información, Especialización en Edición de Publicaciones, así como el inicio de la Maestría en Ciencia de la Información, la aprobación de la profesionalización del programa de Tecnología en Archivística, la asesoría y el acompañamiento a procesos de definición de la política pública en lectura y bibliotecas, la participación en los programas de Estímulo al Talento Estudiantil y Jóvenes Investigadores, y la gestión de recursos financieros mediante los contratos de asesoría y consultoría.

Las debilidades que persisten en relación con profesores y estudiantes son de carácter estructural; es decir, tienen correspondencia con las condiciones propias del sistema de educación del país. No obstante, la Escuela ha implementado diversos mecanismos para mejorar cuantitativa y cualitativamente su planta docente, promoviendo y apoyando la formación en maestría y doctorado, tanto de los profesores vinculados como de los ocasionales, y la asistencia 
a eventos nacionales e internacionales; ha gestionado comisiones administrativas, de estudio y años sabáticos a los que tienen derecho los docentes y ha participado en las convocatorias públicas para la vinculación docente, mecanismos que han contribuido a dinamizar la investigación y la publicación y que son factores determinantes para lograr el incremento salarial de los docentes.

Ha disminuido la deserción de los estudiantes y su tránsito por el plan de estudios es más ágil como consecuencia de los diversos programas que ofrece Bienestar Universitario y de la asesoría de la Jefatura de Formación Académica para favorecer su permanencia. Las directivas no han descuidado el llamado a la participación de los estudiantes en los diferentes órganos académico-administrativos.

La falta de flexibilidad en el plan de estudios es una debilidad que se está superando gracias al diseño de estrategias que permiten vincular a los estudiantes de las tres últimas versiones curriculares con los cursos comunes de Tecnología en Archivística, la promoción de la participación en el programa Sígueme, el reconocimiento de cursos electivos aprobados en otras dependencias y los nuevos cursos electivos profesionales. Asimismo, la programación de horarios, la integración de tecnologías para favorecer la virtualidad y la asesoría personalizada a los estudiantes para cumplir su plan de estudios son elementos que contribuyen a la flexibilidad del programa.

Se conservan y consolidan las fortalezas del programa identificadas en los informes anteriores: permanencia de la RIB (Revista Interamericana de Bibliotecología) en la categoría A2 de Publindex y del Grupo de Investigación en Información, Conocimiento y Sociedad en la categoría A de Colciencias; incremento de la investigación y las publicaciones, de las asesorías y consultorías; continuidad del programa de Estímulo al Talento Estudiantil y participación en el programa Jóvenes Investigadores; evaluación permanente del currículo, expresada en las versiones curriculares cuatro y cinco; reconocimiento de la Escuela y de los profesores en los ámbitos local y nacional, representado en las distinciones Premio Luis Florén (ASEIBI), Premio Rubén Pérez Ortiz (ASCOLBI) y Orden al Mérito Juan del Corral (por el Concejo de Medellín) y la alta formación de los profesores (maestría y doctorado). Finalmente, una de las principales fortalezas resaltada por el CNA se relaciona con la administración y gestión del programa, que durante el periodo evaluado se consolidó y fortaleció, como lo evidencian los planes de acción anual y sus correspondientes informes de gestión.

La realización de acciones innovadoras como la obtención del registro calificado del programa por segunda vez, el comienzo de la Especialización en Edición de Publicaciones, el inicio de la primera cohorte de la Maestría en Ciencia de la Información, con 12 estudiantes de diversas áreas del 
conocimiento (ingeniería, bibliotecología, comunicación social, nutrición y dietética); la asesoría y el acompañamiento a procesos de definición de la política pública en lectura (CERLALC, Plan Nacional de Lectura y Bibliotecas, Municipio de Medellín, Parques Biblioteca); el desarrollo del programa Estímulo al Talento Estudiantil; la participación en el programa Jóvenes Investigadores de la Vicerrectoría de Investigación de la Universidad de Antioquia y la capacidad de autofinanciación del programa son todos logros del mismo.

En consonancia con los lineamientos del Consejo Nacional de Acreditación y con los resultados del proceso de autoevaluación, se concluye que el programa de Bibliotecología tiene un alto grado de calidad: 4.1 sobre 5.0, alcanzado a partir de la superación del $100 \%$ de las debilidades encontradas en el proceso de autoevaluación en 2004; se espera el mantenimiento de sus fortalezas y la realización de acciones innovadoras para seguir construyendo la EIB en una sociedad cada día más exigente, en entornos más competitivos, interconectados y cambiantes.

\section{Evaluación externa}

La evaluación externa expresa que los factores autoevaluados por la EIB tienen globalmente un porcentaje de cumplimiento "en alto grado". Este juicio valorativo es soportado en el señalamiento de algunas fortalezas, como las siguientes:

El equipo de docentes es de muy alto nivel, se ha logrado aumentar la planta [...]. Se registra una fuerte formación en maestría y doctorado [... ]. El tema de Investigación y Extensión es bastante sólido y con un potencial de generación de conocimiento muy alto que puede ser mucho más fuertemente aprovechado en términos de generación de recursos y sostenibilidad para la misma Escuela. Tienen grupos de investigación en categoría A de Colciencias con publicaciones. Se evidencia el incremento de la investigación, la continuidad de la Revista Interamericana de Bibliotecología (RIB) en la categoría A2 de Publindex, la participación docente en eventos nacionales e internacionales como ponentes, además de la producción intelectual traducida en publicaciones [...]. La comunidad académica está muy fortalecida y cohesionada reflejándose un ambiente de confianza y seguridad bastante amplio, entre estudiantes, profesores y personal administrativo [...]. Se proyectan acciones a corto y mediano plazo que fortalecerán todos los aspectos relacionados con Docencia, Investigación y Administración de la EIB (Informe de pares evaluadores externos, julio de 2012).

\section{Evaluación final y reconocimiento público de la calidad}

En esta etapa, el Consejo Nacional de Acreditación de Colombia, luego de estudiar y analizar la información contenida en los informes de los procesos de 
autoevaluación y de evaluación por pares externos, emitió un concepto definitivo que envió al Ministerio de Educación de Colombia, instancia que finalmente otorga, en este caso, la renovación de la Acreditación de Alta Calidad del Programa de Bibliotecología. En consecuencia, se emitió la Resolución 14957 del 19 de noviembre de 2012, mediante la cual se otorga la renovación de la Acreditación de Alta Calidad válida por ocho años para este programa y con base en aspectos como la pertinencia, la interdisciplinariedad y la flexibilización del plan de estudios, el desarrollo de competencias investigativas en los estudiantes, el fortalecimiento del cuerpo profesoral, el incremento de la productividad académica y el trabajo cooperativo con comunidades académicas en el ámbito local, nacional e internacional, así como con los colectivos gremiales de la profesión y el Grupo de Investigación "Información, Conocimiento y Sociedad", clasificado en la categoría A de Colciencias; la Revista Interamericana de Bibliotecología clasificada en la categoría A2 de Publindex y el reconocimiento de la Escuela y de sus profesores con premios otorgados por ASEIBI, ASCOLBI y autoridades gubernamentales locales.

No obstante haber obtenido la renovación de la Acreditación de Alta Calidad, el programa de Bibliotecología debe enfocar su atención en acciones de mejoramiento orientadas a fortalecer el cuerpo profesoral, así como las investigaciones centradas en el objeto de estudio del programa, la movilidad nacional e internacional de doble vía tanto de docentes como de estudiantes y las relaciones con los egresados; y deberá buscar un mejor equilibrio entre la actividad administrativa y académica de los docentes, promover la participación del estudiantado en órganos decisorios del programa, mejorar los planes de transición entre diversas versiones curriculares, optimizar la calidad de la dotación tecnológica del programa y reducir aún más el fenómeno de la deserción.

\section{Conclusiones}

Los procesos de acreditación se constituyen en componente fundamental de las dinámicas de evaluación y mejoramiento continuo de las instituciones de educación superior. En Colombia estos procesos se empiezan a introducir luego de ser aprobada la Ley 30 de Educación Superior (1992). En ella se estimula la introducción de procesos de mejoramiento continuo y de aseguramiento de la calidad, situación que asume el país de manera muy comprometida y que lo ubica como uno de los más destacados en el desarrollo de estos nuevos paradigmas de autorregulación institucional.

Las dinámicas de acreditación en Colombia se llevan a cabo con base en metodologías y procedimientos establecidos por el CNA, ente gubernamental 
encargado de orientar y liderar los procesos de evaluación con fines de acreditación en las diferentes instituciones de educación superior del país. En consecuencia, las etapas que conforman los procesos de acreditación, tales como la autoevaluación, la evaluación por pares externos y la evaluación final, se constituyen en una interesante actividad de reflexión y revisión del hacer académico-administrativo tanto de la institución como de sus unidades académicas, que ofertan programas de formación profesional. Es la oportunidad para identificar fortalezas y debilidades, así como para introducir acciones significativas e innovadoras que contribuyan al mejoramiento y modernización de estos propósitos.

En 1997 la EIB empieza un proceso que le permite reconocerse a sí misma y ante el país como la principal escuela de formación bibliotecológica. Como resultado, en 1999 obtiene, de parte del Ministerio de Educación Nacional/ CNA, la acreditación del programa de Bibliotecología por un periodo de cuatro años; luego en 2004 se le otorga por la misma instancia la re-acreditación por siete años y en el año 2012 obtiene la acreditación de alta calidad por un periodo de ocho años

La Escuela Interamericana de Bibliotecología ha estado a la vanguardia de los procesos de aseguramiento de la calidad con fines de acreditación en América Latina, procesos que la ubican como institución de reconocida trayectoria en la formación de profesionales idóneos y de proyección para la sociedad y como referente importante en estos procesos de calidad; además que le han permitido generar dinámicas de mejoramiento curricular, de cualificación docente, de fomento y fortalecimiento de la actividad investigativa, así como de producción académica y para el mantenimiento de la infraestructura física y de los equipos necesarios para el desarrollo del Programa.

El tránsito exitoso por tres procesos de autoevaluación y acreditación ponen a la EIB de cara a una primera autoevaluación internacional como reto para un nuevo periodo de aseguramiento de alta calidad. En cuanto a los resultados obtenidos en estos tres procesos, es evidente el avance y la calidad de la Escuela en términos curriculares, de investigación, de acciones para la permanencia de los estudiantes, de mejoramiento de los procesos de administración y gestión curricular y, sobre todo, de fomento a la formación de los docentes en maestría y doctorado. Igualmente cuentan a su favor la permanencia de acciones de calidad desde la primera acreditación como la publicación en revistas científicas, la participación en eventos nacionales e internacionales y el autosostenimiento con procesos de asesorías y consultorías, como quedó consignado a lo largo del artículo, y donde además se puede apreciar la compatibilidad de valoraciones tanto a nivel interno (autoevaluación) como externo (pares evaluadores) y el juicio final sobre el estado del Programa (Consejo Nacional de Acreditación, Ministerio de Educación Nacional). 
En general, los procesos de autoevaluación y acreditación han permitido a la EIB dinamizar los procesos curriculares, desarrollar la planeación con base en planes establecidos en las autoevaluaciones que le dan un carácter estratégico de largo plazo y lo dejan ejecutar re-estructuraciones (administrativas y curriculares) tendientes a la modernización del plan de estudios y del Proyecto Educativo Institucional. Pero la Escuela ha conocido también algunas debilidades relacionadas con la escasa participación de los egresados en el devenir de la EIB, la falta de sistematización permanente en las acciones de mejoramiento y la necesidad de contar con un plan efectivo de comunicaciones con las audiencias que conforman la comunidad académica. Igualmente, este proceso reta a la Escuela a emprender acciones encaminadas hacia la acreditación internacional.

\section{REFERENCIAS BIBLIOGRÁFICAS}

Arias Cruz, Álvaro y Cárdenas Lilia, (2012), Informe de evaluación externa con fines de acreditación, Bogotá: Consejo Nacional de Acreditación, 49 pp.

Ministerio de Educación Nacional (2010), Sistema de aseguramiento de la calidad de la educación superior, Bogotá. Disponible en: http:// www.mineducacion.gov.co/1621/w3-article-235585.html (Fecha de consulta: 13 de agosto de 2012).

Ministerio de Educación Nacional, Consejo Nacional de Acreditación (2006a), Lineamientos para la acreditación de programas, Bogotá, 128 pp.

—_ (2006b), Guía para la renovación de la acreditación de programas académicos de pregrado. Guia de procedimientos no. 4, Bogotá, $11 \mathrm{pp}$.

Roa Valero, Alberto (2002), Hacia un modelo de aseguramiento de la calidad en la educación superior en Colombia: estándares básicos y acreditación de excelencia, Bogotá: Consejo Nacional de Acreditación, 13 pp. Disponible en: http://cms-static.colombiaaprende.edu.co/ cache/binaries/articles-186502_doc_academico8.pdf?binary_rand= 4068 (Fecha de consulta: 4 de julio de 2012).

Universidad de Antioquia, Comité Central de Autoevaluación (2006), Guía de autoevaluación: factores, características e indicadores, Medellín.

Universidad de Antioquia, Escuela Interamericana de Bibliotecología (2011), Informe de autoevaluación para renovar la acreditación del programa de bibliotecología, Medellín, $105 \mathrm{pp}$. 